\title{
Editorial
}

\section{Artificial Neural Networks in Agriculture}

\author{
Sebastian Kujawa *,+(i) and Gniewko Niedbała *,+(i) \\ Department of Biosystems Engineering, Faculty of Environmental and Mechanical Engineering, \\ Poznań University of Life Sciences, Wojska Polskiego 50, 60-627 Poznań, Poland \\ * Correspondence: sebastian.kujawa@up.poznan.pl (S.K.); gniewko.niedbala@up.poznan.pl (G.N.) \\ † These authors contributed equally to this work.
}

Citation: Kujawa, S.; Niedbała, G. Artificial Neural Networks in Agriculture. Agriculture 2021, 11, 497. https://doi.org/10.3390/

agriculture 11060497

Received: 20 May 2021

Accepted: 25 May 2021

Published: 27 May 2021

Publisher's Note: MDPI stays neutral with regard to jurisdictional claims in published maps and institutional affiliations.

Copyright: (c) 2021 by the authors. Licensee MDPI, Basel, Switzerland. This article is an open access article distributed under the terms and conditions of the Creative Commons Attribution (CC BY) license (https:// creativecommons.org/licenses/by/ $4.0 /)$.

\begin{abstract}
Artificial neural networks are one of the most important elements of machine learning and artificial intelligence. They are inspired by the human brain structure and function as if they are based on interconnected nodes in which simple processing operations take place. The spectrum of neural networks application is very wide, and it also includes agriculture. Artificial neural networks are increasingly used by food producers at every stage of agricultural production and in efficient farm management. Examples of their applications include: forecasting of production effects in agriculture on the basis of a wide range of independent variables, verification of diseases and pests, intelligent weed control, and classification of the quality of harvested crops. Artificial intelligence methods support decision-making systems in agriculture, help optimize storage and transport processes, and make it possible to predict the costs incurred depending on the chosen direction of management. The inclusion of machine learning methods in the "life cycle of a farm" requires handling large amounts of data collected during the entire growing season and having the appropriate software. Currently, the visible development of precision farming and digital agriculture is causing more and more farms to turn to tools based on artificial intelligence. The purpose of this Special Issue was to publish high-quality research and review papers that cover the application of various types of artificial neural networks in solving relevant tasks and problems of widely defined agriculture.
\end{abstract}

Keywords: yield prediction; crop models; soil and plant nutrition; automated harvesting; model application for sustainable agriculture; precision agriculture; remote sensing for agriculture; decision supporting systems; neural image analysis; convolutional neural networks

\section{Introduction}

Modern agriculture needs to have a high production efficiency combined with a high quality of obtained products. This applies to both crop and livestock production. To meet these requirements, advanced methods of data analysis are more and more frequently used, including those derived from artificial intelligence methods. Artificial neural networks (ANNs) are one of the most popular tools of this kind. They are widely used in solving various classification and prediction tasks. For some time they have also been used in the broadly defined field of agriculture. They can form part of the precision farming and decision support systems. Artificial neural networks can replace the classical methods of modeling, and are one of the main alternatives to classical mathematical models. The spectrum of the applications of artificial neural networks is very wide. For a long time now, researchers from all over the world have been using these tools to support agricultural production, making it more efficient and providing the highest-quality products possible. The purpose of this Special Issue was to publish high-quality research and review papers that cover the application of various types of artificial neural networks in solving relevant tasks and problems of what is widely defined as agriculture. The Special Issue covers 14 peer-reviewed research papers and 1 review paper. 


\section{Papers in the Special Issue}

In the studies described in the first article [1], the following four features were adopted to develop artificial neural networks to identify grain weevil in wheat kernels: mass, equivalent diameter, humidity, and hardness. The authors developed a total of 100 such models. They concluded that the model based on radial basis function, and the structure of 4:10:1, was optimal in solving the problem of the weevil identification. This model was characterized by the RMSE for the test set at a level of 0.25 .

In the studies presented in the second article [2], artificial neural networks were adopted to examine the impact of the variety and weather conditions on the concentration of ferulic acid, deoxynivalenol, and nivalenol in winter wheat grain. As a result of the studies, three independent neural models were developed, one for each of the parameters considered. These models were based on the multilayer perceptron topology with two hidden layers and 14 inputs. On the basis of sensitivity analyses conducted for the models, the authors pointed out that the experiment variant and wheat variety were two of the most important features determining the concentration of ferulic acid, deoxynivalenol, and nivalenol in winter wheat grain.

The authors of the studies discussed in the third article [3] proposed an ANN-based continual classification method that may be used in solving many potential classification tasks in the field of agriculture. The method is based on memory storage and retrieval, and it combines a convolutional neural network $(\mathrm{CNN})$ and generative adversarial network (GAN). The evaluation of this method was carried out for two classification problems: identification of crop pests and plant leaves. The method was also compared with the regular $\mathrm{CNN}$ approach. The results show that the regular CNNs classify the categories clearly for single task problems, whereas in the continual tasks, they are prone to forgetting problems and cannot balance new and old tasks. The method proposed by the authors has the ability to accumulate knowledge and alleviate forgetting. Therefore it can better distinguish all categories from both old and new tasks.

In the studies described in the fourth article [4], the authors made an attempt to apply artificial neural networks to model the dynamic responses of plant growth as affected by a change in root zone temperature in hydroponic chili pepper plants. They used a non-linear autoregressive model with exogenous input (NARX) for this purpose. The performance of the developed model was evaluated by comparing the estimated dynamic response of plant growth values with the observed values. The RMSE and $\mathrm{R}^{2}$ calculated during the evaluation were $0.49 \mathrm{~g}$ and 0.99 , respectively. The authors assumed that the applied type of neural network was useful in identifying the complex process analyzed.

The authors of the studies presented in the fifth article [5] designed neural network models that were used to estimate the corn grain yield. The inputs of the models were: six vegetation indices (NDVI, NDRE, WDRVI, EXG, TGI, VARI), canopy cover, and plant density. The authors also analyzed the relative importance of the predictor variables. Crop information was acquired using an unmanned aerial vehicle (UAV). It was assumed that the spectral information acquired using remote sensors mounted on UAVs and its further processing in vegetation indices, canopy cover, and plant density allows the characterization and estimation of corn grain yield.

The studies discussed in the sixth article [6] were aimed to develop an accurate spectral model to evaluate the cultivated land quality (CLQ) based on the gross primary productivity (GPP) spectral indicator at different growth stages of late rice. The authors compared three types of models that were used for this purpose-linear model based on partial least squares regression (PLSR), and two non-linear models based on support vector regression (SVR), and genetic algorithm-based backpropagation model neural network (GA-BPNN). The CLQ measurements from 294 training samples and the corresponding GPP values were also used to develop the models. The results presented in the paper show that the GA-BPNN model was more accurate in evaluating CLQ than the other two models.

In the studies presented in the seventh article [7], a hybrid feature extraction procedure to address the feature extraction problem in machine learning models was proposed. 
The procedure combines correlation-based feature selection (CFS) and random forest recursive feature elimination (RF-RFE). The proposed method was tested by implementing it in the development of paddy crop yield models that were based on the following machine learning algorithms: random forests, decision trees, and gradient boosting. The authors assumed that the validation of the described hybrid feature extraction method was profoundly satisfying. They also pointed out that the method may also be used in combination with artificial neural networks for agricultural applications.

In the eighth review article [8], all applications and potential applications of non-linear machine learning (ML) algorithms in classical and in vitro-based plant breeding methods have been discussed. Classification of genetic diversity, yield component analysis, yield stability and genotype $\times$ environment interaction $(G \times E)$ analysis, biotic and abiotic stress assessment, and predicting the outcome of mating designs and hybrid breeding programs through machine learning algorithms has been presented in the first part of the review. Applications of machine learning algorithms in in vitro regeneration studies, including optimizing basal culture media, finding the best combination(s) and interaction of medium additives and conditions, and predicting the outcome of in vitro-based plant biotechnology techniques such as artificial polyploidy induction and Agrobacterium-mediated gene transformation have been discussed in the second part of the review. In the third part of the review, the authors presented the application of ML in high-throughput phenotyping and precision agriculture.

The purpose of this study [9] was to develop a model using artificial neural networks to predict soybean harvest area, yield, and production compared with classical methods of time series analysis. The authors collected data from a time series (1961-2016) regarding soybean production in Brazil to perform the model and the classic methods. The results indicate that artificial neural networks are the best approach to predict soybean harvest area and production, while the classical linear function remains more effective to predict soybean yield. Furthermore, artificial neural networks present as a reliable model to predict time series and can help the stakeholders to anticipate the world soybean offer. Finally, the study shows that artificial neural networks can be effective in predicting a commodities index, even using a short time series, which in this case was 50 years. Research results are an important part of supporting grain and cereal production. Among them, soybean is sixth by production volume and fourth by both production area and economic value. The production is concentrated in Brazil and the USA, which are responsible for more than $70 \%$ of world production.

Study [10] proposed a weakly supervised paddy rice mapping approach based on long short-term memory (LSTM) network and dynamic time warping (DTW) distance. Standard temporal synthetic aperture radar (SAR) backscatter profiles for each land cover type were constructed. Weak samples were then labeled on the basis of their DTW distances to the standard temporal profiles. A time-series feature set was then created that combined multispectral Sentinel-2 bands and Sentinel-1 SAR vertical received (VV) band. Experiments showed that weakly supervised learning outperformed supervised learning in paddy rice identification when field samples were insufficient. With only $10 \%$ of field samples, weakly supervised learning achieved better results in producer's accuracy (0.981 to 0.904) and user's accuracy ( 0.961 to 0.917 ) for paddy rice. Training with $50 \%$ of field samples also presented minor improvement. Finally, a paddy rice map was generated with the weakly supervised approach trained on field samples and DTW-labeled samples. The proposed approach based on DTW distance can reduce field sampling cost since it requires fewer field samples. Validation results indicated that the proposed LSTM classifier is suitable for paddy rice mapping.

In the eleventh article [11], the relationship between soil electrical parameters (apparent soil electrical conductivity (ECa) and magnetic susceptibility (MS) measured at two depths, 0.5 and $1 \mathrm{~m}$ ) and soil compaction was investigated with the use of artificial neural networks. A soil's compaction influences the yield; therefore, the delimitation of management zones connected with this parameter is very important in modern agriculture. 
As the geospatial measurement of soil electrical parameters is reliable and economic, it can be a useful technique for the delimitation of management zones. The neural models of the highest quality were developed with the use of RBF neural networks for the prediction of soil compaction measured for soil layers at $0-0.5$ and $0.4-0.5 \mathrm{~m}$ based on electrical parameters of the soil. The authors highlighted that both electrical parameters, ECa and MS, should be used in the process of the delimitation of management zones. This approach can be employed in precision agriculture, e.g., for the reduction in fuel consumption by variable-depth tillage technology based on soil compaction mapping.

Research article [12] describes the challenges date palm growers face in manual processes such as picking, grading, and packing. The authors highlighted that the main challenges are misclassification of dates, maturity level, size, and texture. This article assesses the CNN architectures: VGG-16, VGG-19, ResNet-50, ResNet-101, ResNet-152, AlexNet, Inception $\mathrm{V} 3$, and $\mathrm{CNN}$ from scratch, considering the learning transfer technique for their training and five hyperparameters to reduce time and improve accuracy in the selection process. The data set they used contains 1002 images corresponding to mature and immature dates in trays. The main results showed that the model obtained from the VGG-19 architecture with a batch of 128 and Adam optimizer with a learning rate of 0.01 presented the best performance with a precision of $99.32 \%$. Therefore, the authors suggest the possibility of using the VGG-19 model to build computer vision systems that help producers improve their classification process.

The thirteenth paper [13] presented the results of a laboratory study about the average degree of coverage and the coverage unevenness coefficient. The research was conducted with a special spray track machine, which functioned as a self-propelled field sprayer. Three artificial plants as three replicates were placed on the machine's route. Water-sensitive papers were attached to artificial plants to form specific surfaces. Four types of nozzles were selected for testing: standard, air-induction single flat fan, anti-drift, and air-induction dual flat fan. The following parameters of nozzles' work were used for the research: pressure, height of boom, spray angle, perpendicular to the ground, and driving speed. The research was conducted with a constant dose of liquid. Based on the research results, it was found that the highest average coverage was obtained for single standard flat fan nozzles and dual anti-drift flat fan nozzles. At the same time, the highest values of unevenness were observed for these nozzles. Inverse relationships were obtained for air-induction nozzles.

In study [14], an alternative method for oil palm tree management is proposed by applying high-resolution imagery, combined with faster-RCNN, for the automatic detection and health classification of oil palm trees. This study used a total of 4172 bounding boxes of healthy and unhealthy palm trees, constructed from $2000 \times 2000$ pixel images. Of the total dataset, $90 \%$ was used for training, and 10\% was prepared for testing using ResNet-50 and VGG-16. Three techniques were used to assess the models' performance: model training evaluation, evaluation using visual interpretation, and ground sampling inspections. The study identified three characteristics needed for detection and health classification: crown size, color, and density. The optimal altitude to capture images for detection and classification was determined to be $100 \mathrm{~m}$. For oil palm tree detection, healthy tree identification, and unhealthy tree identification, ResNet-50 obtained F1 scores of $95.09 \%, 92.07 \%$, and $86.96 \%$, respectively, with respect to visual interpretation ground truth and $97.67 \%, 95.30 \%$, and $57.14 \%$, respectively, with respect to ground sampling inspection ground truth. ResNet-50 yielded better F1 scores than VGG-16 in both evaluations.

The research [15] presents an alternative to the use of pesticides in agriculture and horticulture for weed control. Weeds are a major yield-limiting factor in food production, and the use of pesticides levies heavy costs on human and environmental health. The proposed autonomous weeding robot uses artificial neural networks to detect weeds and plants, following it up with the desired action to restrict the growth of weeds. During the training phase, three types of plants were used to assess the efficiency of the robot in identification and detection tasks. The study reported the highest classification accuracy of $95 \%$ for plant specimens and $99 \%$ for weed specimens. The mean average precision 
(mAP) in object detection was found to be $31 \%$. An urgent need for such alternative weed management solutions in view of the planned gradual phasing out of pesticides was expressed by farmers in northern Germany in conversation with the authors. It is pertinent to mention here the goal set by the EU's planned Organic Action Plan, which envisages at least $25 \%$ of the EU's agricultural land under organic (pesticide-free) farming by 2030.

\section{Conclusions}

The papers presented in this Special Issue cover a wide range of applications of various types of artificial neural networks in agriculture. We hope that these papers will stimulate further research in this domain.

Author Contributions: All editors equally contributed to organizing the Special Issue, to editorial work, and to writing this editorial. All authors have read and agreed to the published version of the manuscript.

Funding: This research received no external funding.

Institutional Review Board Statement: Not applicable.

Informed Consent Statement: Not applicable.

Data Availability Statement: No new data were created or analyzed in this study. Data sharing is not applicable to this article.

Acknowledgments: We thank the authors for submitting manuscripts of high quality and their willingness to further improve them after peer review, the reviewers for their careful evaluations aimed at eliminating weaknesses and their suggestions to optimize the manuscripts and the editorial staff of MDPI for the professional support and the rapid actions taken when necessary throughout the editorial process.

Conflicts of Interest: The authors declare no conflict of interest.

\section{References}

1. Boniecki, P.; Koszela, K.; Świerczyński, K.; Skwarcz, J.; Zaborowicz, M.; Przybył, J. Neural Visual Detection of Grain Weevil (Sitophilus granarius L.). Agriculture 2020, 10, 25. [CrossRef]

2. Niedbała, G.; Kurasiak-Popowska, D.; Stuper-Szablewska, K.; Nawracała, J. Application of Artificial Neural Networks to Analyze the Concentration of Ferulic Acid, Deoxynivalenol, and Nivalenol in Winter Wheat Grain. Agriculture 2020, 10, 127. [CrossRef]

3. Li, Y.; Chao, X. ANN-Based Continual Classification in Agriculture. Agriculture 2020, 10, 178. [CrossRef]

4. Aji, G.K.; Hatou, K.; Morimoto, T. Modeling the Dynamic Response of Plant Growth to Root Zone Temperature in Hydroponic Chili Pepper Plant Using Neural Networks. Agriculture 2020, 10, 234. [CrossRef]

5. García-Martínez, H.; Flores-Magdaleno, H.; Ascencio-Hernández, R.; Khalil-Gardezi, A.; Tijerina-Chávez, L.; Mancilla-Villa, O.R.; Vázquez-Peña, M.A. Corn Grain Yield Estimation from Vegetation Indices, Canopy Cover, Plant Density, and a Neural Network Using Multispectral and RGB Images Acquired with Unmanned Aerial Vehicles. Agriculture 2020, 10, 277. [CrossRef]

6. Zhu, M.; Liu, S.; Xia, Z.; Wang, G.; Hu, Y.; Liu, Z. Crop Growth Stage GPP-Driven Spectral Model for Evaluation of Cultivated Land Quality Using GA-BPNN. Agriculture 2020, 10, 318. [CrossRef]

7. Elavarasan, D.; Vincent, P.M.D.R.; Srinivasan, K.; Chang, C.-Y. A Hybrid CFS Filter and RF-RFE Wrapper-Based Feature Extraction for Enhanced Agricultural Crop Yield Prediction Modeling. Agriculture 2020, 10, 400. [CrossRef]

8. Niazian, M.; Niedbała, G. Machine Learning for Plant Breeding and Biotechnology. Agriculture 2020, 10, 436. [CrossRef]

9. Abraham, E.R.; Mendes dos Reis, J.G.; Vendrametto, O.; Oliveira Costa Neto, P.L.d.; Carlo Toloi, R.; Souza, A.E.d.; Oliveira Morais, M.d. Time Series Prediction with Artificial Neural Networks: An Analysis Using Brazilian Soybean Production. Agriculture 2020, 10, 475. [CrossRef]

10. Wang, M.; Wang, J.; Chen, L. Mapping Paddy Rice Using Weakly Supervised Long Short-Term Memory Network with Time Series Sentinel Optical and SAR Images. Agriculture 2020, 10, 483. [CrossRef]

11. Pentoś, K.; Pieczarka, K.; Serwata, K. The Relationship between Soil Electrical Parameters and Compaction of Sandy Clay Loam Soil. Agriculture 2021, 11, 114. [CrossRef]

12. Pérez-Pérez, B.D.; García Vázquez, J.P.; Salomón-Torres, R. Evaluation of Convolutional Neural Networks' Hyperparameters with Transfer Learning to Determine Sorting of Ripe Medjool Dates. Agriculture 2021, 11, 115. [CrossRef]

13. Cieniawska, B.; Pentos, K. Average Degree of Coverage and Coverage Unevenness Coefficient as Parameters for Spraying Quality Assessment. Agriculture 2021, 11, 151. [CrossRef] 
14. Yarak, K.; Witayangkurn, A.; Kritiyutanont, K.; Arunplod, C.; Shibasaki, R. Oil Palm Tree Detection and Health Classification on High-Resolution Imagery Using Deep Learning. Agriculture 2021, 11, 183. [CrossRef]

15. Shah, T.M.; Nasika, D.P.B.; Otterpohl, R. Plant and Weed Identifier Robot as an Agroecological Tool Using Artificial Neural Networks for Image Identification. Agriculture 2021, 11, 222. [CrossRef] 